\title{
Terahertz Radiation Induced Spin Photocurrents in Non-Magnetic Low Dimensional Structures
}

\author{
S.D. Ganichev and W. Prettl \\ Fakultät für Physik, Universität Regensburg, 93040 Regensburg, Germany

\begin{abstract}
Spin photocurrents generated by homogeneous excitation with terahertz radiation in low dimensional structures are reviewed. Three microscopic mechanisms are responsible for the occurrence of an electric current linked to a uniform spin polarization in a quantum well, the circular photogalvanic effect, the spin-galvanic effect and the magneto-gyrotropic effect. These phenomena provide several methods to investigate various spin properties in low dimensional structures like details of band structure and spin relaxation time.
\end{abstract}

PACS numbers: 72.40.+w, 72.25.Fe, 78.67.-n, 73.21.Fg

\section{Introduction}

Lately, there is much interest in the use of the spin of carriers in semiconductor quantum well (QW) structures together with their charge to realize novel concepts like spintronics [1] and spin-optoelectronics. The transport of the spin of charge carriers in semiconductor nanostructures is one of the key problems in this field. The necessary conditions to realize spintronic devices are high spin polarization in QWs and a large spin-splitting of subbands in $\boldsymbol{k}$-space. The latter is important for the ability to control spins with an external electric field by the Rashba effect [2]. In the paradigmatic spin transistor the electron spins, injected into a two-dimensional electron system, are controllably rotated during their passage from source to drain by means of the Rashba spin-orbit coupling. Significant progress has been achieved recently in generating large spin polarizations, in demonstrating the Rashba splitting and also in using the splitting for manipulating the spins [1]. At the same time as these conditions are fulfilled and spins are polarized in-plane of QW, it has been shown that the spin polarization 
itself drives a current [3]. Recently it has been shown that optical orientation of carriers in zinc-blende based semiconductor nanostructures results in a spin polarized current caused by a new class of optoelectronic effects: spin photocurrents $[4,5]$. Spin photocurrents generated by excitation with circularly polarized radiation in QWs have attracted a growing attention in the recent decade. Several mechanisms of electric currents driven by optically generated spin polarization are observed in zinc-blende-structure based bulk semiconductors and QWs. Among these effects are: the circular photogalvanic effect (CPGE) [6], spin photocurrents due to quantum interference of one- and two-photon excitations (QUIC) [7, 8], the spin-galvanic effect (SGE) [3, 9], the "spin-battery" which applies the photovoltaic effect in $p-n$ junctions [10] and the magneto-gyrotropic effect (MGE) [11].

Homogeneous excitation by circularly polarized infrared radiation of gy-

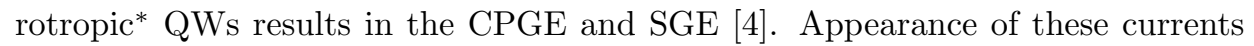
demonstrates a new property of the electron spin in a homogeneous and spin-polarized two-dimensional electron gas (2DEG): its ability to drive an electric current if some general symmetry requirements are met. On a microscopical level the current flow is driven by an asymmetric distribution of spin polarized carriers in $\boldsymbol{k}$-space of systems with lifted spin degeneracy due to $\boldsymbol{k}$-linear terms in the Hamiltonian. The absorption of circularly polarized light results in optical spin orientation due to the transfer of the angular momentum of photons to electrons of a 2DEG. In quantum wells belonging to one of the gyrotropic crystal classes a non-equilibrium spin polarization of uniformly distributed electrons causes a directed motion of electron in the plane of the QW. These effects can be described by simple analytical expressions derived from a phenomenological theory. The requirement of gyrotropy rules out effects depending on the helicity of the radiation field in bulk optically non-active materials like bulk zinc-blende and diamond structure crystals. The reduction of dimensionality as realized in QWs makes spin photocurrents possible. A characteristic feature of this electric current, which occurs in unbiased samples, is that it reverses its direction upon changing the radiation helicity from left-handed to right-handed and vice versa. The circular photogalvanic effect is caused by the asymmetry of the momentum distribution of carriers excited in optical transitions which are sensitive to the light circular polarization due to selection rules [6]. The spin-galvanic effect is a result of spin relaxation and in general does not need optical excitation; however it may also occur due to optical spin orientation [3, 9]. Most recently observed inverse spin galvanic effect predicted in $[12,13]$ demonstrates that in gyrotropic non-magnetic QWs electric current always results in a spin orientation approaching few percents at low temperatures [14-17].

In an external magnetic field spin photocurrents in gyrotropic low dimensional structures may be generated even by unpolarized radiation as it has been

\footnotetext{
${ }^{*}$ The gyrotropic point group symmetry makes no difference between components of axial and polar vectors.
} 
proposed for bulk gyrotropic crystals $[18,19]$. The magneto-gyrotropic effect appears in the presence of an external in-plane magnetic field and is caused by $\boldsymbol{k}$-linear terms in electron-phonon interaction resulting in asymmetric excitation or asymmetric energy relaxation of heated by $\mathrm{THz}$ radiation spin polarized electron gas [11]. This results show that, without a magnetic field, non-equilibrium free carrier heating can be accompanied by spin flow i.e. in pure spin currents.

Several aspects raised by investigation of spin photocurrents are directly connected with the rapidly developing field aimed to novel concepts of semiconductor devices like spintronics and several schemes of quantum computation [1]. Indeed, the necessary conditions to create spintronic devices are high spin polarizations in low dimensional structures and large spin splitting of subbands in $\boldsymbol{k}$-space due to $\boldsymbol{k}$-linear terms in the Hamiltonian which allow to manipulate spins with an external electric field by the Rashba effect [2] (for reviews see [4, 20]). Spin photocurrents offer an experimental access to their investigation. First of all, SGE gives an easy and direct method for investigation of the Rashba-Dresselhaus spin splitting of size quantized subbands [21]. Furthermore, spin photocurrents allow investigation of spin relaxation processes [22, 23].

While spin photocurrents may occur at visible excitation there is a particular interest in spin photocurrents generated by terahertz radiation. This is mostly caused by two reasons. First of all, in the terahertz range spin photocurrents may be observed and investigated much easier than in the visible range where strong spurious photocurrents due to other mechanisms like the Dember effect, photovoltaic effects at contacts etc. mask the relatively weak spin photocurrents. Secondly, in contrast to conventional methods of optical spin orientation using inter-band transitions [24], terahertz radiation excites only one type of charge carriers yielding monopolar spin orientation [4, 25-27]. A substantial portion of investigations of the spin lifetime in semiconductor devices are based on optical spin orientation by interband excitation and further tracing the kinetics of polarized photoluminescence [1]. These studies give important insights into the mechanisms of spin relaxation of photoexcited free carriers. In contrast to these methods of optical spin orientation, monopolar spin orientation allows to study spin relaxation without electron-hole interaction and exciton formation in the conditions close to the case of electric spin injection. Finally, spin photocurrents provide methods to conclude on the in-plane symmetry of QWs and has also been utilized to develop fast detectors to determine the degree of circular polarization of a radiation beam.

\section{Samples and experimental technique}

The experiments were carried out on GaAs, InAs, asymmetric SiGe QWs, GaN QWs, and BeZnMnSe QW structures belonging to two different classes of symmetry. Higher symmetric structures were (001)-oriented QWs which, as our measurements showed, corresponded to the point group $C_{2 v}$. Structures of the lower symmetry class $C_{s}$ were (113)-oriented and asymmetric (110)-oriented QWs. 
Samples of $n$ - and $p$-type QWs with width $L_{w}$ from $7 \mathrm{~nm}$ to $20 \mathrm{~nm}$ and free-carrier densities from $10^{11} \mathrm{~cm}^{-2}$ to $2 \times 10^{12} \mathrm{~cm}^{-2}$ were studied.

For optical excitation mid-infrared (MIR), terahertz, and visible laser radiation was used. Most of the measurements were carried out in the infrared with photon energies smaller than the energy gap $\varepsilon_{\mathrm{g}}$. Depending on the photon energy and $\mathrm{QW}$ band structure the MIR and $\mathrm{THz}$ radiation induce direct transitions between size quantized subbands or, at longer wavelength, Drude absorption. A pulsed TEA- $\mathrm{CO}_{2}$ laser and a molecular THz laser $[28,29]$ have been used as radiation sources in the spectral range between $9.2 \mu \mathrm{m}$ and $496 \mu \mathrm{m}$. Some experiments in the MIR have been carried out making use of the tunability of the free-electron laser FELIX [30]. For optical inter-band excitation a cw-Ti:sapphire laser was used providing radiation with $\lambda=0.777 \mu \mathrm{m}$ and radiation power $P \approx 100 \mathrm{~mW}$.

The circular polarization has been obtained using a Fresnel rhomb, $\lambda / 4$ plates, and a photoelastic modulator for $\mathrm{MIR}, \mathrm{THz}$, and visible radiation, respectively. The helicity $P_{\text {circ }}$ of the incident light was varied from -1 (left-handed circular, $\sigma_{-}$) to +1 (right-handed circular, $\sigma_{+}$) according to $P_{\text {circ }}=\sin 2 \varphi$, where the phase angle $\varphi$ is the angle between the initial plane of polarization and the optical axis of the polarizer. Samples were studied in the temperature range of $4.2 \mathrm{~K}$ to $300 \mathrm{~K}$. The photocurrent $j_{x}$ was measured in the unbiased structures via the voltage drop across a $50 \Omega$ load resistor in a closed circuit configuration [31] (see Fig. 1c). The current in the case of excitation with visible radiation was recorded by a lock-in amplifier in phase with the photoelastic modulator.

Some experiments on the spin-galvanic effect and magneto-gyrotropic effect required an external magnetic field. These measurements were performed at room temperature in a conventional electromagnet with the magnetic field up to $1 \mathrm{~T}$ and at $4.2 \mathrm{~K}$ using a superconducting split-coil magnet with $B$ up to $3 \mathrm{~T}$.

\section{Circular photogalvanic effect}

The CPGE appears due to the asymmetry of the momentum distribution of photoexcited carriers in homogeneous samples and belongs to the class of photogalvanic effects (for reviews see $[4,5,32,33]$ ). The microscopic origin of this current is the conversion of photon angular momentum into directed motion of carriers. The experimental data can be described by simple analytical expressions derived from a phenomenological theory which shows that the effect can only be present in gyrotropic media. This requirement rules out effects depending on the helicity of the radiation in non-optically active materials.

\subsection{Experiment: general features}

With illumination of QW structures by polarized radiation a current signal proportional to the helicity $P_{\text {circ }}$ has been observed in unbiased samples [31]. The signal follows to the temporal structure of the applied $100 \mathrm{~ns}$ laser pulses and reverses its sign by switching the polarization from $\sigma_{+}$to $\sigma_{-}$(see Fig. 1). The 

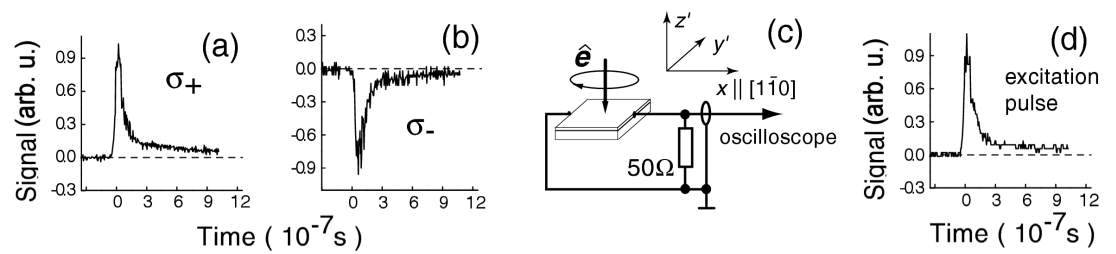

Fig. 1. Oscilloscope traces obtained for pulsed excitation of (113)-grown $n$-type GaAs QWs at $\lambda=10.6 \mu \mathrm{m}$ and normal incidence. Parts (a) and (b) show CPGE signals, (c) the measurement arrangement and (d) a signal pulse of a fast photon drag detector. For (001)-grown QWs oblique incidence was used in order to obtain helicity dependent current.
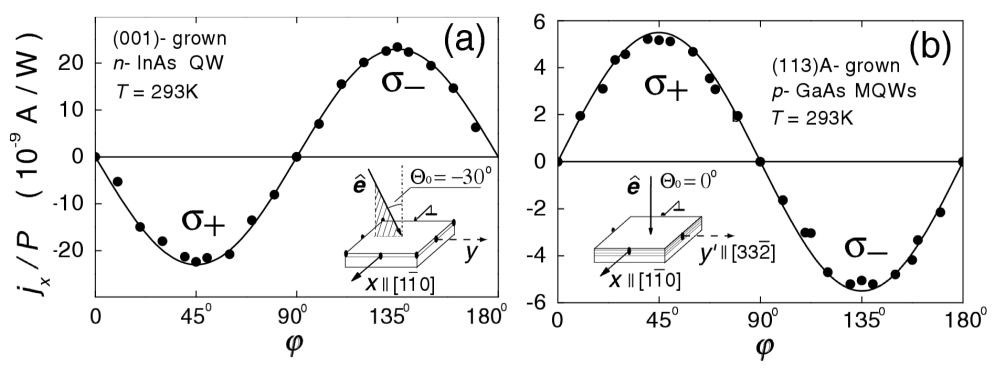

Fig. 2. Photocurrent in QWs normalized by the light power $P$ as a function of the phase angle $\varphi$ defining helicity. Measurements are presented for $T=293 \mathrm{~K}$ and $\lambda=$ $76 \mu \mathrm{m}$. (a) Oblique incidence of radiation with an angle of incidence on (001)-grown QWs (symmetry class $C_{2 v}$ ). (b) Normal incidence of radiation on (113)-grown QWs (symmetry class $C_{s}$ ). Full lines show ordinate scale fits after Eqs. (2) and (3) for the top and lower panel, respectively. The insets show the geometry of the experiment.

radiation induced current and its characteristic helicity dependence shown in Fig. 2 reveals that we are dealing with the CPGE.

In (001)-oriented samples a helicity dependent signal is only observed under oblique incidence [6]. A variation of the angle of incidence $\Theta_{0}$ in the incidence plane around $\Theta_{0}=0^{\circ}$ changes the sign of the current. For light propagating along $\langle 110\rangle$ direction the photocurrent flows perpendicular to the wave vector of the incident light (see Fig. 2a). For illumination along a cubic axis $\langle 100\rangle$ both a transverse and a longitudinal CPGE current are detected [4]. In samples grown on a (113)-GaAs surface or on (001)-miscut substrates representing the lower symmetry class $C_{s}$, the CPGE has been observed also under normal incidence of radiation [6] as shown in Fig. 2b. The current does not change its sign by the variation of $\Theta_{0}$ and assumes its maximum at $\Theta_{0}=0^{\circ}$. This is in contrast to (001)-oriented samples and in accordance to the phenomenological theory of the CPGE for $C_{s}$. For normal incidence in this symmetry the current always flows along the [1 $\overline{1} 0]$ -direction perpendicular to the plane of mirror reflection of the point group $C_{s}$. 


\subsection{Phenomenology}

Phenomenologically the CPGE current $\boldsymbol{j}$ can be described as (see e.g. [5])

$$
j_{\lambda}=\sum_{\mu} \gamma_{\lambda \mu} \mathrm{i}\left(\boldsymbol{E} \times \boldsymbol{E}^{*}\right)_{\mu},
$$

where $\boldsymbol{\gamma}$ is a pseudo-tensor, $\boldsymbol{E}$ is the complex amplitude of the radiation electric field, i $\left(\boldsymbol{E} \times \boldsymbol{E}^{*}\right)_{\mu}=\hat{e}_{\mu} E_{0}^{2} P_{\text {circ }}, E_{0}$ and $\hat{\boldsymbol{e}}$ are the electric field amplitude and the unit vector pointing in the direction of light propagation, respectively. In general, in addition to the CPGE current given in Eq. (1), two other photocurrents can be present simultaneously, namely the linear photogalvanic effect (LPGE) and the photon drag effect [5]. Both effects were observed in low dimensional structures (for review see [4]). These photocurrents are not changed in sign or amplitude if the polarization is switched from $\sigma_{+}$to $\sigma_{-}$which allow to distinguish them from the CPGE. They do not require spin orientation and are outside the scope of the present investigation.

In the following we analyze Eq. (1) for symmetries relevant to experiment. Hereafter we use for (001)-grown QWs Cartesian coordinates $x \|$ [110], $y \|$ [110], $z \|[001]$ and for (113)-grown QWs the coordinates $x^{\prime}=x\left\|[1 \overline{1} 0], y^{\prime}\right\|[33 \overline{2}]$, and $z^{\prime} \|$ [113]. For $C_{2 v}$ symmetry the photocurrent is given by

$$
j_{x}=\gamma_{x y} \hat{e}_{y} E_{0}^{2} P_{\text {circ }}, \quad j_{y}=\gamma_{y x} \hat{e}_{x} E_{0}^{2} P_{\text {circ }} .
$$

If $\hat{e}$ is along $\langle 110\rangle$, then the current flows normal to the light propagation direction. If the sample is irradiated with $\hat{\boldsymbol{e}}$ parallel to $\langle 100\rangle$ the current is neither parallel nor perpendicular to the light propagation direction. Another conclusion from Eq. (2) is that in QWs of $C_{2 v}$ symmetry the photocurrent can only be induced under oblique incidence of radiation. For normal incidence $\hat{\boldsymbol{e}}$ is parallel to [001] and hence the current vanishes. In contrast to this result in QWs of $C_{s}$ symmetry a photocurrent also occurs for normal incidence of the radiation because the tensor $\gamma$ has an additional component $\gamma_{x z^{\prime}}$. The current here is given by

$$
j_{x}=\left(\gamma_{x y^{\prime}} \hat{e}_{y^{\prime}}+\gamma_{x z^{\prime}} \hat{e}_{z^{\prime}}\right) E_{0}^{2} P_{\text {circ }}, \quad j_{y^{\prime}}=\gamma_{y^{\prime} x} \hat{e}_{x} E_{0}^{2} P_{\text {circ }} .
$$

At normal incidence, $\hat{e}_{x}=\hat{e}_{y^{\prime}}=0$ and $\hat{e}_{z^{\prime}}=1$, the current in the QW flows along $x$, i.e. perpendicular to the mirror reflection plane. The dependence of the photocurrent on the angle of incidence $\Theta_{0}$ is determined by the value of the projection $\hat{\boldsymbol{e}}$ on the $x$ - (y-) axis (see Eqs. (2)) or on the $z^{\prime}$-axis (Eqs. (3)). The phenomenological picture outlined above perfectly describes the experimental observations [4].

\section{3. $C P G E$ at inter-band transitions}

The CPGE at inter-band transitions is most easily conceivable from the schematic band structure shown in Fig. 3 [6]. Microscopically, a conversion of photon helicity into a spin photocurrent arises due to $\boldsymbol{k}$-linear terms in the effective Hamiltonian $\hat{H}=\sum_{l m} \beta_{l m} \sigma_{l} k_{m}$ where $\boldsymbol{k}$ is the electron wave vector, $\sigma_{l}$ are 

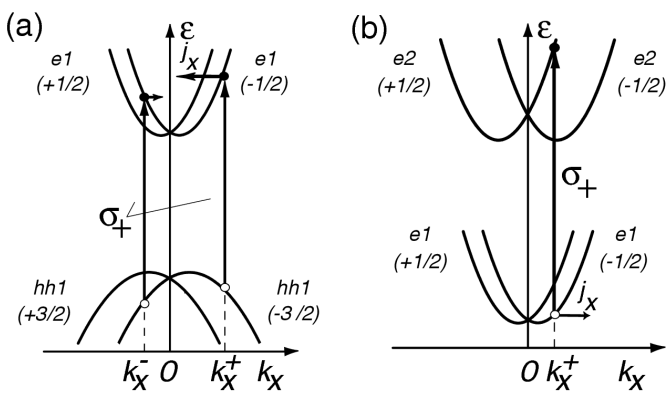

Fig. 3. Microscopic picture of the CPGE at direct transitions in $C_{s}$ point group for (a) inter-band transitions and (b) for transitions between size quantized subbands in the conduction band. Currents are shown for one subband only.

the Pauli spin matrices and $\beta_{l m}$ are real coefficients. The coefficients $\beta_{l m}$ form a pseudo-tensor subjected to the same symmetry restriction as the transposed pseudo-tensor $\boldsymbol{\gamma}$. The sources of $\boldsymbol{k}$-linear terms are the bulk inversion asymmetry (BIA) also called the Dresselhaus term [34] (including a possible interface inversion asymmetry [35]) and possibly a structural inversion asymmetry (SIA) usually called the Rashba term [2].

For the sake of simplicity we take into account a band structure consisting only of the lowest conduction subband e1 and the highest heavy-hole subband hh1 whose energy dispersion is described by $\varepsilon_{\mathrm{e} 1, \pm 1 / 2}(\boldsymbol{k})=\left[\left(\hbar^{2} k_{x}^{2} / 2 m_{\mathrm{e} 1}\right) \pm \beta_{\mathrm{e} 1} k_{x}+\varepsilon_{\mathrm{g}}\right]$ and $\varepsilon_{\mathrm{hh} 1, \pm 3 / 2}(\boldsymbol{k})=-\left[\left(\hbar^{2} k_{x}^{2} / 2 m_{\mathrm{hh} 1}\right) \pm \beta_{\mathrm{hh} 1} k_{x}\right]$, respectively.

For absorption of circularly polarized radiation of photon $\hbar \omega$ energy and momentum conservation allow transitions only for two values of $k_{x}$. We consider a $\mathrm{QW}$ of $C_{s}$ symmetry where due to selection rules the optical transitions occur from $m_{s}=-3 / 2$ to $m_{s}=-1 / 2$ for right-handed polarized light and from $m_{s}=3 / 2$ to $m_{s}=1 / 2$ for left-handed polarized light. Here $m_{s}$ are the spin quantum numbers of the electron states. The corresponding transitions for, for instance, $\sigma_{+}$photons occur at

$$
k_{x}^{ \pm}=+\frac{\mu}{\hbar^{2}}\left(\beta_{\mathrm{e} 1}+\beta_{\mathrm{hh} 1}\right) \pm \sqrt{\frac{\mu^{2}}{\hbar^{4}}\left(\beta_{\mathrm{e} 1}+\beta_{\mathrm{hh} 1}\right)^{2}+\frac{2 \mu}{\hbar^{2}}\left(\hbar \omega-E_{\mathrm{g}}\right)},
$$

and are shown in Fig. 3a by the solid vertical arrows. Here $\mu=\left(m_{\mathrm{e} 1} m_{\mathrm{hh} 1}\right) /$ $\left(m_{\mathrm{e} 1}+m_{\mathrm{hh} 1}\right)$. The "center of mass" of these transitions is shifted from the point $k_{x}=0$ by $\left(\beta_{\mathrm{e} 1}+\beta_{\mathrm{hh} 1}\right)\left(\mu / \hbar^{2}\right)$. Thus the sum of the electron velocities in the excited states in the conduction band is non-zero resulting in a spin polarized net current. Switching the polarization from $\sigma_{+}$to $\sigma_{-}$mirrors the picture and the current direction reverses. The microscopic theory of CPGE in QWs for inter-band excitation was worked out in $[36,37]$.

The circular photogalvanic effect at interband transitions has been observed most recently in GaAs QWs [38, 39]. At visible excitation strong spurious photocurrent due to other mechanisms like the Dember effect, photovoltaic effects at 
contacts etc. mask the relatively weak CPGE. However, application of polarization selective measurements, like modulation of polarization allows to extract the CPGE current. In the infrared range, where effects mentioned above vanish, detection of the CPGE caused in this case by inter- or intra-subband transition does not require modulation of polarization.

\subsection{CPGE at inter-subband transitions}

For direct transition between size quantized states in the conduction or valence band the model is very similar to inter-band transitions discussed above [40]. In Fig. 3b we sketch the situation for QWs of $C_{s}$ symmetry. Due to selection rules optical transitions for monochromatic, say $\sigma_{+}$, radiation occur only at a fixed $k_{x}^{+}$ where the energy of the incident light matches the transition energy as is indicated by the arrow in Fig. 3b. Therefore optical transitions induce an imbalance of momentum distribution in both subbands yielding an electric current in $x$ direction with contributions from e1 and e2. As in $n$-type QWs the energy separation $\varepsilon_{21}$ between e1 and e2 is typically larger than the energy of longitudinal optical phonons $\hbar \omega_{\mathrm{LO}}$, the non-equilibrium distribution of electrons in e2 relaxes rapidly due to emission of phonons. As a result, the momentum relaxation time in e2 subband, $\tau_{p}^{(2)}$, is much smaller than $\tau_{p}^{(1)}$, the momentum relaxation time in e1 subband. Thus the current is mainly due to the photogenerated holes in the initial state of the resonant optical transition in the e1 subband.

The microscopic theory of CPGE for direct inter-subband transitions in $n$-type QWs for both $C_{s}$ and $C_{2 v}$ symmetry was developed in [40] with the result that the current is proportional to the derivative of the absorbance. For $\tau_{p}^{(2)}$ much less than $\tau_{p}^{(1)}$ it was shown that for $C_{s}$ symmetry

$$
j_{x} \sim\left(\beta_{y x}^{(2)}+\beta_{y x}^{(1)}\right)\left(\tau_{p}^{(1)}-\tau_{p}^{(2)}\right) \frac{\mathrm{d} \eta_{12}(\hbar \omega)}{\mathrm{d} \hbar \omega} I P_{\text {circ }} \hat{e}_{y},
$$

where $\beta_{y x}^{(1)}$ and $\beta_{y x}^{(2)}$ are components of $\boldsymbol{\beta}$ in the e1 and e2 subbands, respectively. The change of sign of the photocurrent with photon energy may be understood from Fig. 3b. It is seen that at large photon energy, $\hbar \omega>\varepsilon_{21}$, excitation occurs at positive $k_{x}$ resulting in a current $j_{x}$ shown by arrow in Fig. 3b. Decreasing of the photon frequency shifts the transition towards negative $k_{x}$ and reverses the current direction.

Similar arguments hold for $C_{2 v}$ symmetry under oblique incidence. However, in contrast to spin-flip processes occurring for $C_{s}$ symmetry described above, in $C_{2 v}$ symmetry due to selection rules the absorption of circularly polarized radiation is spin-conserving [5]. For these symmetry the CPGE is also proportional to the derivative of absorption and is given by

$$
j_{x} \sim\left(\beta_{y x}^{(2)}-\beta_{y x}^{(1)}\right)\left(\tau_{p}^{(1)}-\tau_{p}^{(2)}\right) \frac{\mathrm{d} \eta_{12}(\hbar \omega)}{\mathrm{d} \hbar \omega} I P_{\text {circ }} \hat{e}_{y} .
$$

Since the CPGE in QW structures of $C_{2 v}$ symmetry is caused by spin-conserving 
optical transitions, the photocurrent described by Eq. (6) in contrast to Eq. (5) is proportional to the difference of subband spin splitting.

Experimentally CPGE at resonant transitions was observed in $n$-type GaAs samples of QW widths from 8.2 to $8.6 \mathrm{~nm}$. Direct optical transitions between e1 and e2 subband were excited applying MIR radiation of the $\mathrm{CO}_{2}$ laser or free-electron laser FELIX. A current signal proportional to the helicity has been observed at normal incidence in (113)-samples and at oblique incidence in (001)-oriented samples indicating the CPGE [40]. In Fig. 4 the data are presented for a (001)-grown $n$-GaAs QW of $8.2 \mathrm{~nm}$ width. It is seen that in direction $x$ the current for both, $\sigma_{+}$and $\sigma_{-}$radiation, changes sign at a frequency of the absorption peak. Experimental results shown in Fig. 4, in particular the sign inversion of the spectral behaviour of the current, are in a good agreement with microscopic theory developed in [40] (see Eq. (6)).

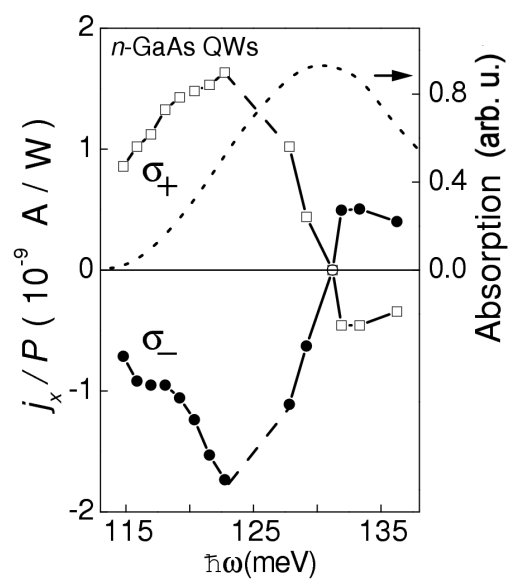

Fig. 4. Photocurrent in QWs normalized by $P$ as a function of the photon energy $\hbar \omega$. Measurements are presented for $n$-type (001)-grown GaAs QWs of $8.2 \mathrm{~nm}$ width (symmetry class $C_{2 v}$ ) at $T=293 \mathrm{~K}$ and oblique incidence of radiation with an angle of incidence $\Theta_{0}=20^{\circ}$. The absorption of the MIR laser radiation results in direct transitions between $\mathrm{e} 1$ and $\mathrm{e} 2$ subbands. The current $j_{x}$ is perpendicular to the direction of light propagation. The dotted line shows the absorption measured using a Fourier spectrometer.

The CPGE at direct inter-subband transitions has also been observed in $p$-type GaAs QWs demonstrating spin orientation of holes (see Fig. 2b) [6, 23]. To achieve hh1-lh1 transitions $\mathrm{THz}$ radiation was applied.

\subsection{CPGE at intra-subband transitions (Drude absorption)}

Now we consider indirect intra-subband transitions. This situation is usually realized in the $\mathrm{THz}$ range where the photon energy is not high enough to excite 
direct inter-subband transitions. Due to energy and momentum conservation intra-subband transitions can only occur by absorption of a photon and simultaneous absorption or emission of a phonon. This process is described by virtual transitions involving intermediate states. It can be shown that transitions via intermediate states within one and the same subband do not contribute to the spin photocurrent. However, spin selective indirect optical transitions excited by circularly polarized light can generate a spin current if virtual processes involve intermediate states in different subbands [6].

Optical absorption caused by indirect transitions in $n$-type samples have been obtained applying $\mathrm{THz}$ radiation covering the range of $76 \mu \mathrm{m}$ to $280 \mu \mathrm{m}$. The experiments were carried out on GaAs [6, 36], InAs [6], semimagnetic ZnBeMnSe [41], and GaN [42] QWs. The energy separation between e1 and e2 size-quantized subbands of those samples is much larger than the $\mathrm{THz}$ photon energies used here. Therefore the absorption is caused by indirect intra-subband optical transitions. With illumination of (001)-grown QWs at oblique incidence of $\mathrm{THz}$ radiation a current signal proportional to the helicity $P_{\text {circ }}$ has been observed (see Fig. 2a) showing that the Drude absorption of a 2D electron gas results in spin orientation and the CPGE. CPGE at intra-subband absorption was also observed in $p$-type samples at long wavelengths [31, 36, 43], where the photon energies are smaller than the energy separation between heavy-hole and light-hole subbands.

\section{6. $C P G E$ in $S i G e Q W s$}

In symmetrical (001)-grown SiGe QWs no CPGE current has been detected as expected from the presence of inversion symmetry in both materials. However, in artificially grown non-symmetric QWs, CPGE has been observed being caused by the Rashba spin-orbit coupling due to a built-in potential gradient in the QWs [43]. CPGE in SiGe QWs has been detected in $p$-type structures at direct heavy-hole-light-hole transitions and at indirect intra-subband transitions.

\section{Spin-galvanic effect}

The picture of the spin photocurrent given so far involved the asymmetry of the momentum distribution of photoexcited carriers, i.e. the CPGE. After momentum relaxation of the photoexcited carriers the CPGE vanishes, however, a spin orientation may still be present if the spin relaxation time is longer than the momentum relaxation time. In such a case an asymmetry of spin-flip scattering of non-equilibrium spin polarized carriers may contribute to the total current. This current is caused by the spin-galvanic effect (SGE) and in general it does not require photoexcitation.

\subsection{Phenomenology}

The SGE is due to spin relaxation of a uniform non-equilibrium spin polarization in QWs of gyrotropic symmetry [3]. Phenomenologically, an electric 
current can be linked to the electron's averaged spin polarization $\boldsymbol{S}$ by

$$
j_{\alpha}=\sum_{\gamma} Q_{\alpha \gamma} S_{\gamma}
$$

Like in the case of CPGE here we have a pseudo-tensor $\boldsymbol{Q}$ with the same symmetry restrictions like $\gamma$. For $C_{2 v}$ symmetry of (001)-grown QWs only two linearly independent components, $Q_{x y}$ and $Q_{y x}$, may be non-zero so that

$$
j_{x}=Q_{x y} S_{y}, \quad j_{y}=Q_{y x} S_{x} .
$$

Hence, SGE current needs a spin component lying in the plane of QWs. In QWs of $C_{s}$ symmetry an additional tensor component $Q_{x z^{\prime}}$ may be non-zero and the SGE may be caused by spins oriented normally to the plane of QW.

\subsection{Microscopic model}

Microscopically, the spin-galvanic effect is caused by asymmetric spin-flip relaxation of spin polarized electrons in systems with $\boldsymbol{k}$-linear contributions to the effective Hamiltonian [3]. Figure 5 sketches the electron energy spectrum along $k_{x}$ with the spin dependent term $\beta_{y x} \sigma_{y} k_{x}$. In this case $\sigma_{y}$ is a good quantum number. Spin orientation in $y$-direction causes the unbalanced population in the subbands. The current flow is caused by $\boldsymbol{k}$-dependent spin-flip relaxation processes. Spins oriented in $y$-direction are scattered along $k_{x}$ from the higher filled, e.g. spin-up subband, $|+1 / 2\rangle_{y}$, to the less filled spin-down subband, $|-1 / 2\rangle_{y}$. Four quantitatively different spin-flip scattering events exist and are sketched in Fig. 5 by bent arrows. The spin-flip scattering rate depends on the values of the
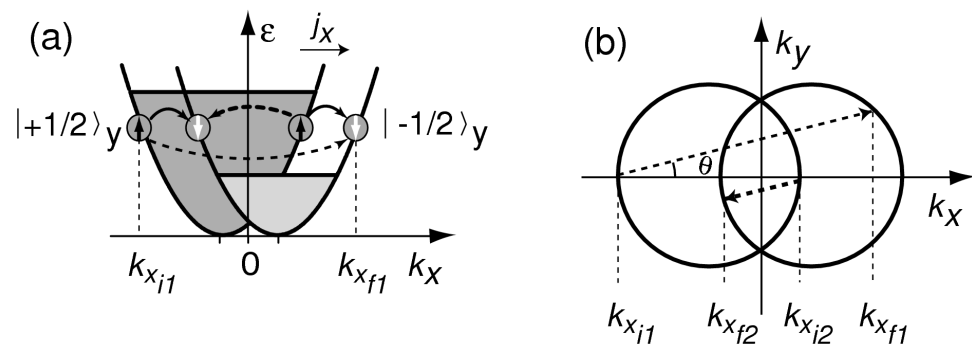

Fig. 5. Microscopic origin of the spin-galvanic current in the presence of $\boldsymbol{k}$-linear terms in the electron Hamiltonian. (a) One-dimensional sketch: the $\sigma_{y} k_{x}$ term in the Hamiltonian splits the conduction band into two parabolas with the spin $m_{s}= \pm 1 / 2$ pointing in the $y$-direction. If one spin subband is preferentially occupied, e.g. by spin injection (the $|+1 / 2\rangle_{y}$-states in the figure) asymmetric spin-flip scattering results in a current in $x$-direction. The rate of spin-flip scattering depends on the value of the initial and final $\boldsymbol{k}$-vectors. Thus transitions sketched by dashed arrows yield an asymmetric occupation of both subbands and hence a current flow. These transitions are also shown in two dimensions (b) by dashed arrows at scattering angle $\theta$. If instead of the spin-down subband the spin-up subband is preferentially occupied, the current direction is reversed. 
wave vectors of the initial and the final states, respectively [44]. Two scattering processes shown by broken arrows are inequivalent and generate an asymmetric carrier distribution around the subband minima in both subbands. This asymmetric population results in a current flow along the $x$-direction. The uniformity of spin polarization in space is preserved during the scattering processes. Therefore the spin-galvanic effect differs from other experiments where the spin current is caused by inhomogeneities [45-47]. Up to now the spin-galvanic effect has been recorded at optical spin orientation caused by inter-band, inter-subband, as well as intra-subband transitions [3, 9]. Let us note that the reverse process to the spin-galvanic effect i.e. a spin polarization induced by an electric current flow has been theoretically considered in $[12,13]$.

\subsection{Microscopic theory}

The microscopic theory of the spin-galvanic effect is presented as follows [9]. The occurrence of a current is due to the spin dependence of the electron scattering matrix elements $M_{\boldsymbol{k}^{\prime} \boldsymbol{k}}$. The $2 \times 2$ matrix $\hat{M}_{\boldsymbol{k}^{\prime} \boldsymbol{k}}$ can be written as a linear combination of the unit matrix $\hat{I}$ and Pauli matrices as follows:

$$
\hat{M}_{\boldsymbol{k}^{\prime} \boldsymbol{k}}=A_{\boldsymbol{k}^{\prime} \boldsymbol{k}} \hat{I}+\boldsymbol{\sigma} \cdot \boldsymbol{B}_{\boldsymbol{k}^{\prime} \boldsymbol{k}}
$$

where $A_{\boldsymbol{k}^{\prime} \boldsymbol{k}}^{*}=A_{\boldsymbol{k} \boldsymbol{k}^{\prime}}, B_{\boldsymbol{k}^{\prime} \boldsymbol{k}}^{*}=B_{\boldsymbol{k} \boldsymbol{k}^{\prime}}$ due to hermiticity of the interaction and $A_{-\boldsymbol{k}^{\prime},-\boldsymbol{k}}=A_{\boldsymbol{k} \boldsymbol{k}^{\prime}}, B_{-\boldsymbol{k}^{\prime},-\boldsymbol{k}}=-B_{\boldsymbol{k} \boldsymbol{k}^{\prime}}$ due to the symmetry under time inversion. The spin-dependent part of the scattering amplitude is given by [44]:

$$
\boldsymbol{\sigma} \cdot \boldsymbol{B}_{\boldsymbol{k}^{\prime} \boldsymbol{k}}=v\left(\boldsymbol{k}-\boldsymbol{k}^{\prime}\right)\left[\sigma_{x}\left(k_{y}^{\prime}+k_{y}\right)-\sigma_{y}\left(k_{x}^{\prime}+k_{x}\right)\right] .
$$

We note that Eq. (10) determines the spin relaxation time, $\tau_{s}^{\prime}$, due to the ElliotYafet mechanism. The spin-galvanic current has the form [9]

$$
j_{\mathrm{SGE}, x}=Q_{x y} S_{y} \sim e n_{\mathrm{e}} \frac{\beta_{y x}^{(1)}}{\hbar} \frac{\tau_{p}}{\tau_{s}^{\prime}} S_{y}, \quad j_{\mathrm{SGE}, y}=Q_{y x} S_{x} \sim e n_{\mathrm{e}} \frac{\beta_{x y}^{(1)}}{\hbar} \frac{\tau_{p}}{\tau_{s}^{\prime}} S_{x} .
$$

Since scattering is the origin of the SGE, the spin-galvanic current is determined by the Elliot-Yafet spin relaxation time. The relaxation time $\tau_{s}^{\prime}$ is proportional to the momentum relaxation time $\tau_{p}$. Therefore the ratio $\tau_{p} / \tau_{s}^{\prime}$ in Eq. (11) does not depend on the momentum relaxation time. The in-plane average spin $S_{x}$ in Eq. (11) decays with the total spin relaxation time $\tau_{s}$. Thus the time decay of the spin-galvanic current following the pulsed photoexcitation is determined by $\tau_{s}$.

\subsection{SGE at optical orientation in the presence of magnetic field}

Excitation of QWs by circularly polarized light results in a spin polarization which, at proper orientation of the electron spins, causes the spin-galvanic effect. Because of the tensor equivalence the spin-galvanic current induced by circularly polarized light always occurs simultaneously with the CPGE. It has been recently shown that at inter-subband transitions the spin-galvanic effect may be separated from CPGE making use of the spectral behaviour at resonance [9]. 
Another possibility to investigate the spin-galvanic effect without contributions of the CPGE to the current has been introduced in [3]. The spin polarization was obtained by absorption of circularly polarized radiation at normal incidence on (001)-grown QWs as depicted in the inset of Fig. 6. For normal incidence the CPGE as well as the spin-galvanic effect vanish because $\hat{e}_{x}=\hat{e}_{y}=0$ (see Eqs. (2)) and $S_{x}=S_{y}=0$ (see Eqs. (8)), respectively. Thus, we have a spin orientation along the $z$ coordinate but no spin photocurrent.

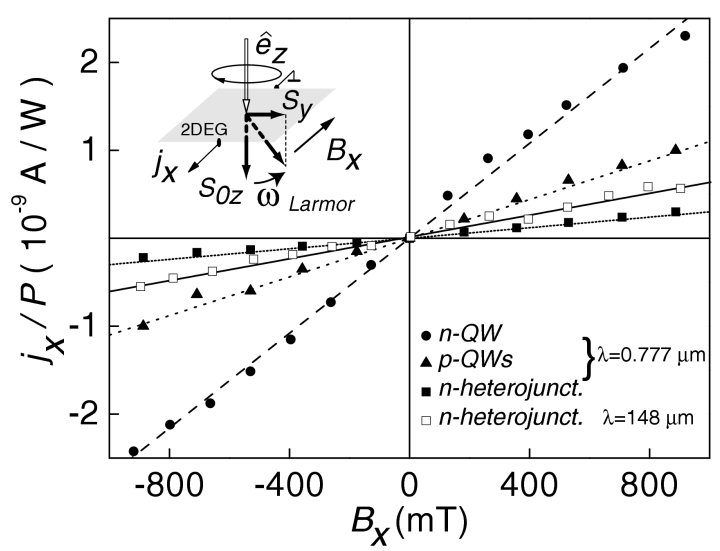

Fig. 6. Spin-galvanic current $j_{x}$ normalized by $P$ as a function of magnetic field $B$ at $T=293 \mathrm{~K}$ for various samples. Data are presented for GaAs/AlGaAs heterostructures at normal incident with circularly polarized radiation of $\lambda=0.777 \mu \mathrm{m}$ with $P=100 \mathrm{~mW}$ and $\lambda=148 \mu \mathrm{m}$ with $P=20 \mathrm{~kW}$. The inset shows the geometry of the experiment.

To obtain an in-plane component of the spins, necessary for the spin-galvanic effect, a magnetic field $\boldsymbol{B} \| x$ has been applied. Due to the Larmor precession a non-equilibrium spin polarization $S_{y}$ is induced being

$$
S_{y}=-\frac{\omega_{\mathrm{L}} \tau_{s \perp}}{1+\left(\omega_{\mathrm{L}} \tau_{s}\right)^{2}} S_{0 z}
$$

where $\tau_{s}=\sqrt{\tau_{s \|} \tau_{s \perp}}, \tau_{s \|}, \tau_{s \perp}$ are the longitudinal and transverse electron spin relaxation times, $\omega_{\mathrm{L}}$ is the Larmor frequency. The denominator in Eq. (12) yielding the decay of $S_{y}$ for $\omega_{\mathrm{L}} \tau_{\mathrm{s}}>1$ is well known from the Hanle effect [24].

Both, for visible and infrared radiation, a current due to SGE has been observed for all (001)-grown $n$-type GaAs and InAs samples after applying an in-plane magnetic field (Figs. 6, 7) $[3,26]$.

For low magnetic fields $B$ where $\omega_{\mathrm{L}} \tau_{s}<1$ holds, the current increases linearly as expected from Eqs. (8) and (12). The polarity of the current depends on the direction of the excited spins determined by the radiation helicity and the direction of the applied magnetic field. For magnetic field applied along $\langle 110\rangle$ the current flows along the magnetic field. For $B \|\langle 100\rangle$ both the transverse and the longitudinal effects are observed [4]. 


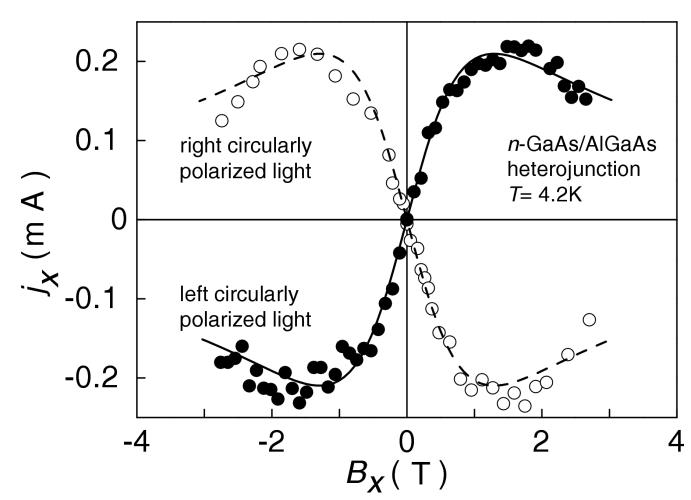

Fig. 7. Spin-galvanic current $j_{x}$ as a function of magnetic field $B$ for normal incident circularly polarized radiation at $\lambda=148 \mu \mathrm{m}$ and radiation power $20 \mathrm{~kW}$. Solid and dashed curves are fitted after Eqs. (8) and (12) using the same value of the spin relaxation time $\tau_{s}$ and scaling of the ordinate.

For higher magnetic fields the current assumes a maximum and decreases upon further increase in $B$, as shown in Fig. 7. This drop of the current is ascribed to the Hanle effect [24]. The experimental data are well described by Eqs. (8) and (12). The observation of the Hanle effect demonstrates that free carrier intra-subband transitions can polarize the spins of electron systems. The measurements allow to obtain the spin relaxation time $\tau_{s}$ from the peak position of the photocurrent where $\omega_{\mathrm{L}} \tau_{s}=1$ holds [3].

\subsection{SGE at optical orientation without external magnetic field}

In the experiments described above an external magnetic field was used for re-orientation of an optically generated spin polarization. The SGE can also occur at optical excitation only, without application of an external magnetic field. The necessary in-plane component of the spin polarization can be obtained by oblique incidence of the exciting circularly polarized radiation but in this case the CPGE may also occur interfering with the SGE.

However, a pure SGE may be obtained at inter-subband transitions in $n$-type GaAs QWs [9]. The spin orientation is generated by spin-selective optical excitation followed by spin-non-specific thermalization. The magnitude of the spin polarization and hence the current depends on the initial absorption strength but not on the $\boldsymbol{k}$ of transition. Thus the spin-galvanic current is proportional to the absorbance [26]. In contrast, as shown above the spectrum of CPGE changes sign and vanishes in the centre of resonance (see Fig. 4 and [40]). Thus, if a measurable helicity dependent current is present in the centre of the resonance, it must be attributed to the SGE.

Experiments have been carried out making use of the spectral tunability of the free electron laser FELIX [30]. The photon energy dependence of the current was measured for incidence in two different planes with components of propagation 
along the $x$ - and $y$-directions (see Fig. 8). It can be seen that for a current along $x$ the spectral shape is similar to the derivative of the absorption spectrum indicating the CPGE as already discussed above. When the sample was rotated by $90^{\circ}$ about $z$ the sign change in the current, now along $y$, disappears and its spectral shape follows more closely the absorption spectrum. The lack of a sign change for current along $y \|[110]$ in the experiment shows that the spin-galvanic effect dominates for this orientation.

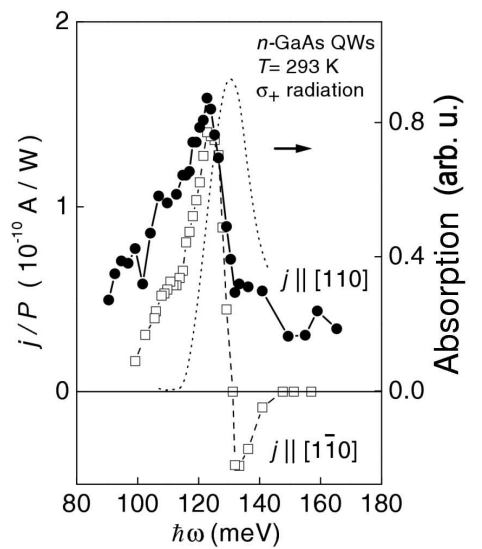

Fig. 8. Photocurrent in QWs normalized by the light power $P$ at oblique incidence of right-handed circularly polarized radiation on $n$-type (001)-grown GaAs/AlGaAs QWs of $8.2 \mathrm{~nm}$ width at $T=293 \mathrm{~K}$ as a function of the photon energy $\hbar \omega$. Circles: current in [110] direction in response to irradiation parallel [110]. Rectangles: current in [110] direction in response to irradiation parallel [110]. Dotted line shows the absorption measured using a Fourier transform spectrometer.

The non-equivalence of the [110]- and [110]-axes is caused by the interplay of BIA and SIA terms in the Hamiltonian. It has been shown in [9] that in directions $y$ and $x$ the SGE and the CPGE are proportional to terms with the sum and the difference of BIA and SIA terms, respectively. For our samples it appears that in the case where they subtract, the CPGE dominates over SGE. Conversely, when BIA and SIA terms add the CPGE is suppressed and the SGE dominates consistent with the lack of sign change for the current along the $y$-direction (see Fig. 8).

\section{Circular photogalvanic effect versus spin-galvanic effect}

The circular photogalvanic effect and the spin-galvanic effect have in common that the current flow is driven by an asymmetric distribution of carriers in $\boldsymbol{k}$-space in systems with lifted spin degeneracy due to $\boldsymbol{k}$-linear terms in the Hamiltonian. The crucial difference between both effects is that the spin-galvanic effect may be 
caused by any means of spin injection, while the CPGE needs optical excitation with circularly polarized radiation. Even if the spin-galvanic effect is achieved by optical spin orientation, as discussed here, the microscopic mechanisms are different. The spin-galvanic effect is caused by asymmetric spin-flip scattering of spin polarized carriers and it is determined by the process of spin relaxation (see Fig. 5). If spin relaxation is absent, the spin-galvanic current vanishes. In contrast, the CPGE is the result of selective photoexcitation of carriers in $\boldsymbol{k}$-space with circularly polarized light due to optical selection rules and depends on momentum relaxation (see Fig. 3b). In some optical experiments the observed photocurrent may represent a sum of both effects. For example, if we irradiate an (001)-oriented QW at oblique incidence of circularly polarized radiation, we obtain both selective photoexcitation of carriers in $\boldsymbol{k}$-space determined by momentum relaxation and the spin-galvanic effect due to an in-plane component of non-equilibrium spin polarization. Thus both effects contribute to the current occurring in the plane of the QW. The two mechanisms can be distinguished by time resolved measurements because usually momentum relaxation time and spin relaxation time are different.

\section{Magneto-gyrotropic effects in quantum wells}

Magneto-photogalvanic effects (MPGE) are photogalvanic currents which occur at polarized or unpolarized optical irradiation due to an external magnetic field. The magnetic field breaks the time inversion symmetry affecting photogalvanic currents and resulting in new mechanisms of current formation. For instance, in inversion asymmetric but non-gyrotropic bulk crystals like GaAs illumination with circular polarized radiation does not yield a current. However, in the presence of an external magnetic field this effect becomes possible and has been detected applying the radiation of a $\mathrm{CO}_{2}$-laser [48]. Essential progress has been achieved in generation of magnetic field induced photogalvanic effects applying near and mid infrared laser radiation (for reviews see [5, 32, 33]).

Decreasing the radiation frequency to the terahertz range makes new mechanisms of excitation possible. The small photon energies yield the access to various kinds of magnetic field induced resonances. In addition, the Drude absorption is enhanced at low frequencies. In bulk materials resonant photocurrents are observed due to free carrier spin-flip transitions and transitions between Zeeman-split impurity levels [49]. These currents are spin polarized.

Further access to spin polarized currents gives the irradiation of gyrotropic low dimensional structures in the presence of a magnetic field. Magneto-gyrotropic effects may have a resonance character due to transitions between RashbaDresselhaus spin split subbands [50-52] as well as being non-resonant at the Drude absorption $[11,53]$. The latter effect is caused by electron gas heating and links spin-optics to electronics, because magneto-gyrotropic effects due to the Drude absorption can also be observed at excitation in the microwave range where the basic mechanism is free carrier absorption as well. 
Like photogalvanic effects in the absence of a magnetic field, the magnitude and direction of magneto-photogalvanic currents depend on the radiation polarization state. However, in addition to polarization of radiation, the orientation of the magnetic field with respect to crystallographic directions plays an important role, especially for low dimensional structures. As a consequence, a proper choice of experimental geometry allows to investigate various possible contributions to MPGE separately. The dependence of the photocurrent properties on polarization, relative orientation of the current flow, magnetic field and crystallographic axes may be obtained from phenomenological theory which does not require knowledge of its microscopic origin. Within the linear approximation in the magnetic field strength $\boldsymbol{B}$, the MPGE current is given by [32]:

$$
j_{\alpha}=\sum_{\beta \gamma \delta} \phi_{\alpha \beta \gamma \delta} B_{\beta}\left\{E_{\gamma} E_{\delta}^{*}\right\}+\sum_{\beta \gamma} \mu_{\alpha \beta \gamma} B_{\beta} \hat{e}_{\gamma} E_{0}^{2} P_{\text {circ }} .
$$

Here the fourth rank pseudo-tensor $\boldsymbol{\phi}$ is symmetric in the last two indices, and $\boldsymbol{\mu}$ is a regular third rank tensor. While the second term requires circularly polarized radiation, the first term may be non-zero even for unpolarized radiation.

Magnetic field induced photocurrents in gyrotropic quantum wells uncover a new type of magneto-photogalvanic phenomena. The gyrotropic point group symmetry makes no difference between components of axial and polar vectors, and hence allows an electric current $\boldsymbol{j} \propto \boldsymbol{I B}$. Photocurrents which require simultaneously gyrotropy and the presence of a magnetic field may be gathered in a class of magneto-optical phenomena denoted as magneto-gyrotropic photogalvanic effects. Such currents are observed in the range of fundamental absorption and at the excitation with intense terahertz radiation. While mechanisms of current due to inter-band excitation are spin independent [54-58], terahertz radiation generates spin polarized currents. THz radiation induced magneto-gyrotropic effects are observed due to direct transitions between spin-split Rashba-Dresselhaus branches of the lowest electron subband [50-52] and non-resonant Drude absorption [11, 53]. All these currents are caused by spin dependent processes and represent spin photocurrents here. Usually spin photocurrents require gyrotropic quantum wells and illumination with circularly polarized radiation. In an external magnetic field even unpolarized radiation yields spin polarized currents $[11,53]$.

Magneto-gyrotropic effects were detected in (001)-oriented QWs based on III-V compounds. Experiments were carried out on asymmetric QWs structures of $C_{2 v}$ symmetry and, therefore, here we will only focus on them.

For this point group, it is convenient to write the components of magneto-photogalvanic current in the coordinate system $(x y z)$. Then for normal incidence of light and an in-plane magnetic field Eq. (13) can be reduced to [53]:

$$
\begin{aligned}
j_{x}= & S_{1} B_{y} E_{0}^{2}+S_{2} B_{y}\left(\left|e_{x}\right|^{2}-\left|e_{y}\right|^{2}\right) E_{0}^{2} \\
& +S_{3} B_{x}\left(e_{x} e_{y}^{*}+e_{y} e_{x}^{*}\right) E_{0}^{2}+S_{4} B_{x} E_{0}^{2} P_{\text {circ }},
\end{aligned}
$$




$$
\begin{aligned}
j_{y}= & S_{1}^{\prime} B_{x} E_{0}^{2}+S_{2}^{\prime} B_{x}\left(\left|e_{x}\right|^{2}-\left|e_{y}\right|^{2}\right) E_{0}^{2} \\
& +S_{3}^{\prime} B_{y}\left(e_{x} e_{y}^{*}+e_{y} e_{x}^{*}\right) E_{0}^{2}+S_{4}^{\prime} B_{y} E_{0}^{2} P_{\text {circ }} .
\end{aligned}
$$

Here the parameters $S_{1}$ to $S_{4}$ and $S_{1}^{\prime}$ to $S_{4}^{\prime}$ are expressed in terms of non-zero components of the tensors $\phi$ and $\boldsymbol{\mu}$ allowed by the $C_{2 v}$ point group [11].

The first terms on the right hand side of Eqs. (14) (containing $S_{1}, S_{1}^{\prime}$ ) yield a current in the QW plane which is independent of radiation's polarization. This current is induced even by unpolarized radiation. Each following contribution has a special polarization dependence which permits to separate it experimentally from others. For linearly polarized light, polarization dependences are caused by the terms described by parameters $S_{2}, S_{2}^{\prime}$ and $S_{3}, S_{3}^{\prime}$. These terms are proportional to $\left|e_{x}\right|^{2}-\left|e_{y}\right|^{2}=\cos 2 \alpha$ and $e_{x} e_{y}^{*}+e_{y} e_{x}^{*}=\sin 2 \alpha$, respectively, where $\alpha$ is the angle between the plane of linear polarization and the $x$ axis. The last terms (parameters $S_{4}, S_{4}^{\prime}$ ), being proportional to $P_{\text {circ }}$, are non-zero for elliptically polarized radiation and vanish for linear polarization.

For elliptically polarized light all contributions are allowed. Elliptically and, in particular, circularly polarized radiation is achieved by passing laser radiation, initially linearly polarized along $x^{\prime}$-axis, through a $\lambda / 4$-plate. Rotation of the plate results in a variation of both, the linear polarization and the helicity as follows:

$$
\begin{aligned}
& P_{\text {lin }}^{\prime} \equiv \frac{1}{2}\left(\left|e_{x}\right|^{2}-\left|e_{y}\right|^{2}\right)=\frac{1+\cos 4 \varphi}{2}, \\
& P_{\operatorname{lin}} \equiv \frac{1}{2}\left(e_{x} e_{y}^{*}+e_{y} e_{x}^{*}\right)=\frac{1}{2} \sin 4 \varphi .
\end{aligned}
$$

Two Stokes parameters $P_{\text {lin }}^{\prime}, P_{\text {lin }}$ describe the degrees of linear polarization and $\varphi$ is the angle between the optical axis of $\lambda / 4$ plate and the direction of the initial polarization $x$.

\subsection{Drude absorption}

The most detailed study of polarization dependences of the magneto-gyrotropic currents were provided in [11]. In InAs and GaAs QW structures, an illumination with terahertz radiation in the presence of an in-plane magnetic field results in a photocurrent in full agreement with the phenomenological theory. All current contributions described by Eqs. (14) were separately observed by the variation of polarization state and measuring the current in different directions relative to the magnetic field orientation and crystallographic axes.

Figures 9 and 10 give two characteristic examples of magneto-gyrotropic effects. Figure 9 shows the data of a magnetic field induced photocurrent perpendicular to $\boldsymbol{B}$ in high mobility InAs QWs samples. The magnetic field dependence is shown for three different polarization states. Neither rotation of the polarization plane of the linearly polarized radiation nor variation of helicity changes the signal 


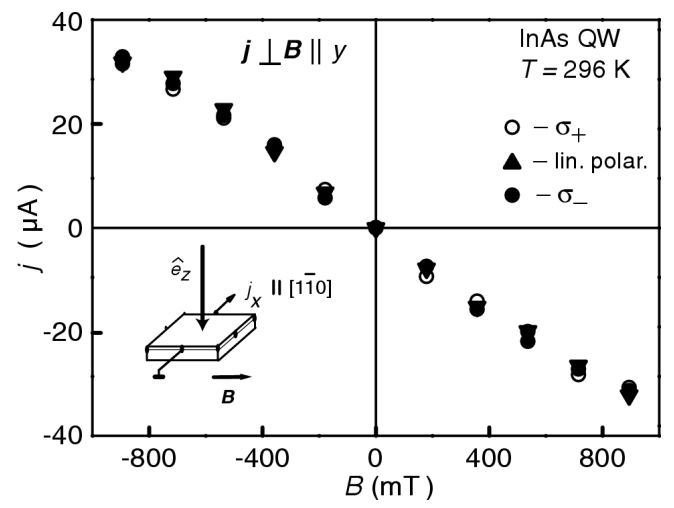

Fig. 9. Magnetic field dependence of the photocurrent measured in InAs QWs structures at room temperature with the magnetic field $\boldsymbol{B}$ parallel to the $y$ axis. Data are given for normal incident optical excitation at the wavelength $\lambda=148 \mu \mathrm{m}$ for linear $(\boldsymbol{E} \| x)$, right-handed circular $\left(\sigma_{+}\right)$, and left-handed circular $\left(\sigma_{-}\right)$polarization. The current is measured in the direction perpendicular to $\boldsymbol{B}$. The inset shows the geometry of the experiment. Data are after [11].

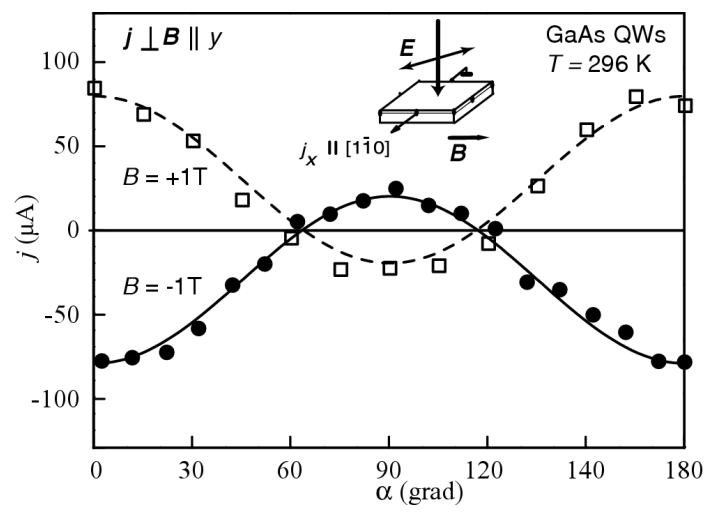

Fig. 10. Photocurrent in GaAs QWs for $\boldsymbol{j} \perp \boldsymbol{B} \| y$ as a function of the azimuth angle $\alpha$. The photocurrent excited by normal incident radiation of $\lambda=148 \mu \mathrm{m}$ is measured at room temperature for magnetic fields in two opposite directions. The broken line and the full line are fitted according to $j \propto \cos 2 \alpha$. This polarization dependence corresponds to the second term in Eq. (14). The inset shows the geometry of the experiment. Data are after [11].

magnitude. This demonstrates that current is independent of polarization. On the other hand, the current changes its direction upon magnetic field reversal. This behaviour is described by $j_{x} \propto I B_{y}$ and corresponds to the first term on the right hand side of Eqs. (14). The absence of a $\varphi$-dependence indicates that the second term in Eqs. (14) is negligibly small. The second example demonstrates a current sensitive to polarization (see Fig. 10). It is observed in the same geometry but in 
another sample, which has low mobility and is homogeneously doped. The measured polarization dependence can doubtlessly be attributed to the second terms in Eqs. (14) [11].

Such a difference in the current properties is caused by the interplay between various microscopical mechanisms responsible for the current generation. Radiation of $\lambda=90 \mu \mathrm{m}$ and $148 \mu \mathrm{m}$ causes a current due to the Drude absorption. The microscopic theory presented in [11] shows that two classes of mechanisms dominate the magneto-gyrotropic effects. The current may be either induced by asymmetry of optical excitation and/or by an asymmetry of relaxation. Though in all cases the absorption is independent of the light's polarization, the photocurrent depends on polarization for the first class of the magneto-gyrotropic effects but is independent of polarization state for the second class.

The first mechanism of current generation in QWs in the presence of a magnetic field is related to the asymmetry of optical excitation. The characteristic feature of this mechanism is a sensitivity to the polarization of light. Free electron absorption requires a momentum transfer from phonons to electrons. A photocurrent induced by these transitions appears due to an asymmetry in $\boldsymbol{k}$-space of either electron-photon or electron-phonon interaction. In gyrotropic media the electron-phonon interaction $\hat{V}_{\text {el-phon }}$ contains, in addition to the main contribution, an asymmetric spin-dependent term $\propto \sigma_{\alpha}\left(k_{\beta}+k_{\beta}^{\prime}\right)$ given by $[11,44,27,59,60]$

$$
\hat{V}_{\mathrm{el}-\mathrm{phon}}\left(\boldsymbol{k}^{\prime}, \boldsymbol{k}\right)=\Xi_{c} \sum_{j} \varepsilon_{j j}+\Xi_{c v} \xi \sum_{j}\left[\left(\boldsymbol{k}^{\prime}+\boldsymbol{v}\right) \times \boldsymbol{\sigma}\right]_{j} \varepsilon_{j+1 j+2} .
$$

Here $\varepsilon_{j j^{\prime}}$ is the phonon-induced strain tensor dependent on the phonon wave vector $\boldsymbol{q}=\boldsymbol{k}^{\prime}-\boldsymbol{k}, \Xi_{c}$ and $\Xi_{c v}$ are the intra-band and inter-band constants of the deformation potential. For zinc-blende-lattice QWs the coefficient $\xi$ is given by [27]:

$$
\xi=\frac{\mathrm{i} \hbar p_{c v}}{3 m_{0}} \frac{\Delta_{\mathrm{so}}}{E_{\mathrm{g}}\left(E_{\mathrm{g}}+\Delta_{\mathrm{so}}\right)},
$$

where $m_{0}$ is the free-electron mass, $E_{\mathrm{g}}$ and $\Delta_{\text {so }}$ are the band gap and spin-orbit splitting of the valence band of the bulk semiconductor used in the QW layer, $p_{c v}=\left\langle S\left|\hat{p}_{z}\right| Z\right\rangle$ is the inter-band matrix element of the momentum operator. Microscopically this asymmetric spin-dependent term in electron-phonon interaction is caused by structural and bulk inversion asymmetry which also cause RashbaDresselhaus band spin splitting in $\boldsymbol{k}$-space.

The asymmetry of electron-phonon interaction results in non-equal rates of indirect optical transitions for opposite wave vectors in each spin subband with $s_{\alpha}= \pm 1 / 2$. This causes an asymmetric distribution of photoexcited carriers within the subband $s_{\alpha}$ and yields therefore a flow, $\boldsymbol{i}_{\alpha}$, of electrons in this subband. This situation is sketched in Fig. 11a for the spin-down subband. The single and double horizontal arrows in this figure indicate the difference in electron-phonon interaction strength for positive and negative wave vectors. The important point now is that single and double arrows are interchanged for the other spin direction (see 
Eq. (17)). The enhancement of the electron-phonon interaction rate for specific $\boldsymbol{k}$-vectors depends on the spin direction. Therefore for the other spin subband, the situation is reversed. This is analogous to the well known spin-orbit interaction where the shift of the $\varepsilon(\boldsymbol{k})$ dispersion depends also on the spin direction. Thus without a magnetic field two equal currents in spin-up and spin-down subbands flow in opposite directions and cancel each other exactly. The non-equilibrium electron distribution in $\boldsymbol{k}$-space is characterized by zero electric current but non-zero pure spin current $\boldsymbol{j}_{\text {spin }}=(1 / 2)\left(\boldsymbol{j}_{1 / 2}-\boldsymbol{j}_{-1 / 2}\right)$. The application of a magnetic field results, due to the Zeeman effect, in different equilibrium populations of the subbands. This is seen in Fig. 11a, where the Zeeman splitting is largely exaggerated to simplify visualization. Currents flowing in opposite directions become non-equivalent resulting in a spin polarized net electric current. Since the current is caused by asymmetry of photoexcitation, it may contribute to all terms and may depend on the polarization of radiation. Therefore, for these mechanisms the photocurrents exhibit a characteristic polarization dependence given by the second and third terms in Eqs. (14) described by the coefficients $S_{2}, S_{2}^{\prime}, S_{3}, S_{3}^{\prime}$.
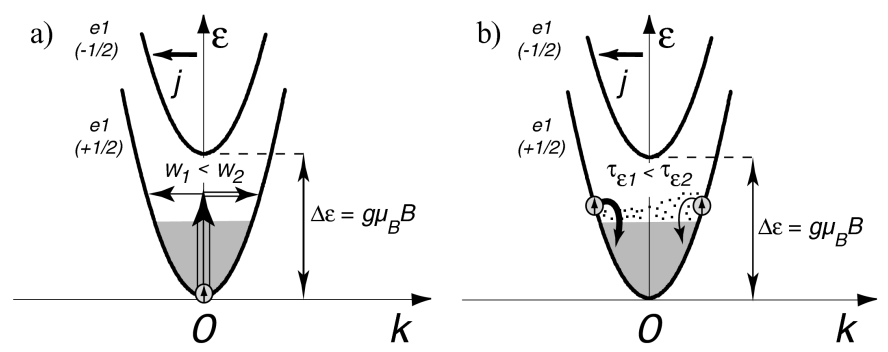

Fig. 11. Model of magneto-gyrotropic effects caused by: (a) asymmetric photoexcitation, (b) asymmetry of energy relaxation. The spin subband $(-1 / 2)$ is preferably occupied due to the Zeeman splitting in an in-plane magnetic field. $W_{1}<W_{2}$ are optical transitions probabilities for positive and negative $k$, respectively. $\tau_{\varepsilon 1}$ and $\tau_{\varepsilon 2}$ are energy relaxation times.

The second mechanism of current formation is caused by the energy and spin relaxation of a non-equilibrium electron gas in gyrotropic systems. Here we give an example of the current formation due to energy relaxation. The Drude absorption by free electrons leads to an electron gas heating, i.e. to a non-equilibrium energy distribution of electrons. It is assumed, for simplicity, that the excitation results in isotropic non-equilibrium occupation. Due to the same asymmetry of electron-phonon interaction discussed for the first mechanism (Eq. (17)), hot electrons with opposite $\boldsymbol{k}$ have different relaxation rates. This situation is sketched in Fig. 11b for a spin-down subband, where two arrows of different thicknesses denote non-equal relaxation rates. Whether $-\boldsymbol{k}$ or $+\boldsymbol{k}$ states relax preferentially, depends on the spin direction. Again the arrows in Fig. 11b need to be interchanged for 
the other spin-subband. The asymmetric relaxation results in an electric current in each subband. For $B=0$ the currents in the spin-up and spin-down subbands have opposite directions and cancel exactly. But a pure spin current flows which accumulates opposite spins at opposite edges of the sample. In the presence of a magnetic field the currents moving in the opposite directions do not cancel due to the unequal population of the spin subbands (see Fig. 11b) and a spin polarized electric current results. The asymmetry of relaxation processes is described only by the first and last terms in Eqs. (14), e.g. by coefficients $S_{1}, S_{1}^{\prime}, S_{4}, S_{4}^{\prime}$.

\section{Application of spin photocurrents}

Spin photocurrents provide an experimental access to spin properties of low dimensional structures and are also implied for detection of helicity of terahertz radiation with picosecond time resolution [61]. Spin photocurrents are applied to investigate spin relaxation times at monopolar spin orientation where only one type of charge carriers is involved in the excitation-relaxation process [4]. This condition is close to that of electrical spin injection in semiconductors. One of these methods based on the Hanle effect in the spin-galvanic current [3] was already introduced above. The other is based on nonlinearity of spin photocurrents allowing to measure spin sensitive bleaching [22, 23].

A further important application of both spin-galvanic and circular photogalvanic effects was addressed by Ganichev et al. in [21]. It is demonstrated that angular dependent measurements of spin photocurrents allow to separate Dresselhaus and Rashba terms. The relative strength of these terms is of importance because it is directly linked to the manipulation of the spin of charge carriers in semiconductors, one of the key problems in the field of spintronics. Spin polarization may be tuned by means of the Rashba spin-orbit coupling in quantum wells. In addition to the Rashba coupling, caused by structural inversion asymmetry, also a Dresselhaus type of coupling, caused by a lack of inversion symmetry in the host material, contributes to spin splitting. In $C_{2 v}$ symmetry these terms are given by symmetric and antisymmetric tensor components $\beta_{\mathrm{BIA}}=(1 / 2)\left(\beta_{x y}+\beta_{y x}\right)$ and $\beta_{\text {SIA }}=(1 / 2)\left(\beta_{x y}-\beta_{y x}\right)$. Dresselhaus term $\beta_{\text {BIA }}$ and Rashba term $\beta_{\text {SIA }}$ result from bulk inversion asymmetry and from structural inversion asymmetry, respectively.

Both, Rashba and Dresselhaus couplings, result in spin splitting of the band and give rise to a variety of spin dependent phenomena which allow to evaluate the magnitude of the total spin splitting of electron subbands. However, usually it is not possible to extract the relative strengths of Rashba and Dresselhaus terms in the spin-orbit coupling. In obtaining the Rashba coefficient, the Dresselhaus contribution is normally neglected. At the same time, Dresselhaus and Rashba terms can interfere in such a way that macroscopic effects vanish though the individual terms are large [44, 62]. For example, both terms can cancel each other resulting in a vanishing spin splitting in certain $\boldsymbol{k}$-space directions [4]. This cancellation 
leads to the disappearance of an antilocalization [63], the absence of spin relaxation in specific crystallographic directions [44, 64], and the lack of Shubnikov-de Haas beating [62]. In [65] the importance of both Rashba and Dresselhaus terms was pointed out: tuning $\beta_{\text {SIA }}$ such that $\beta_{\text {SIA }}=\beta_{\text {BIA }}$ holds, allows to build a non-ballistic spin field-effect transistor.

By mapping the magnitude of the spin photocurrent in the plane of a QW the ratio of both terms can directly be determined from experiment and does not relay on theoretically obtained quantities [21]. Indeed, the spin-galvanic current is driven by the electron in-plane average spin $\boldsymbol{S}_{\|}$according to [21]:

$$
\boldsymbol{j}_{\mathrm{SGE}} \propto\left(\begin{array}{c}
\beta_{\mathrm{BIA}}-\beta_{\mathrm{SIA}} \\
\beta_{\mathrm{SIA}}-\beta_{\mathrm{BIA}}
\end{array}\right) \boldsymbol{S}_{\|},
$$

if $\boldsymbol{S}_{\|}$is aligned along one of the cubic axes. Therefore, the spin-galvanic current $\boldsymbol{j}_{\mathrm{SGE}}$ consists of Rashba and Dresselhaus coupling induced currents, $\boldsymbol{j}_{\mathrm{R}}$ and $\boldsymbol{j}_{\mathrm{D}}$ (see Fig. 12a). Their magnitudes are $j_{\mathrm{R}} \propto \beta_{\mathrm{SIA}}\left|\boldsymbol{S}_{\|}\right|, j_{\mathrm{D}} \propto \beta_{\mathrm{BIA}}\left|\boldsymbol{S}_{\|}\right|$and their ratio is

$$
j_{\mathrm{R}} / j_{\mathrm{D}}=\beta_{\mathrm{SIA}} / \beta_{\mathrm{BIA}} .
$$
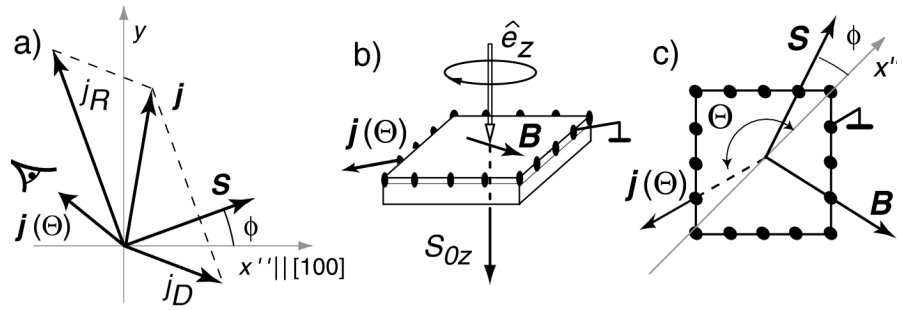

Fig. 12. Angular dependence of the spin-galvanic current (a) and geometry of the experiment ((b) and (c)).

Figure 13 shows the angular dependence of the spin-galvanic current $j_{\mathrm{SGE}}$ measured at room temperature on (001)-oriented $n$-type InAs/ $\mathrm{Al}_{0.3} \mathrm{Ga}_{0.7} \mathrm{Sb}$ single QWs of $15 \mathrm{~nm}$ width. Because of the admixture of helicity independent magneto-gyrotropic effects the spin-galvanic effect is extracted after eliminating current contributions which are helicity independent: $j_{\mathrm{SGE}}=\left(j_{\sigma_{+}}-j_{\sigma_{-}}\right) / 2$.

The non-equilibrium in-plane spin polarization $\boldsymbol{S}_{\|}$is prepared as described above. Circularly polarized light at normal incidence on the QW plane polarizes the electrons in the lowest conduction subband resulting in a monopolar spin orientation in the $z$-direction (Fig. 12b). An in-plane magnetic field $(B=1 \mathrm{~T})$ rotates the spin around the magnetic field axis (precession) and results in a non-equilibrium in-plane spin polarization $S_{\|} \propto \omega_{\mathrm{L}} \tau_{s}$. In the range of the applied magnetic field strength the spin-galvanic current rises linearly with $B$ indicating $\omega_{\mathrm{L}} \tau_{s}<1$ and, thus, the Hanle effect is not present. The angle between the ma- 

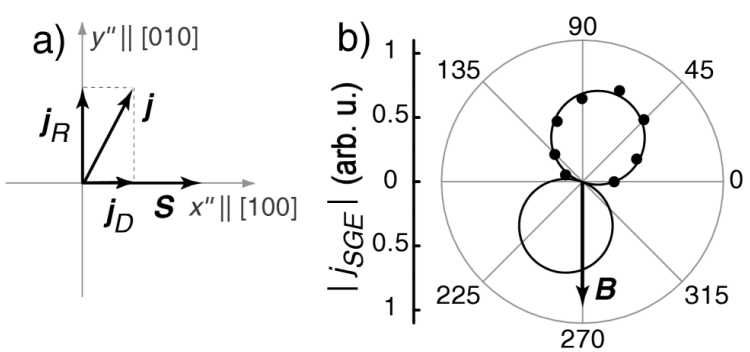

Fig. 13. Photocurrent in $n$-type InAs single QWs. Part (a) indicates relation between spin polarization and current contributions (after Eq. (19)). Part (b) shows measurements of the spin galvanic current as a function of angle $\Theta$. Data are presented in polar coordinates. Data are after [21].

gnetic field and $\boldsymbol{S}_{\|}$in general depends on details of the spin relaxation process. In these InAs QW structures, the isotropic Elliott-Yafet spin relaxation mechanism dominates [44]. Thus the in-plane spin polarization $\boldsymbol{S}_{\|}$of photoexcited carriers is always perpendicular to $\boldsymbol{B}$ and can be varied by rotating $\boldsymbol{B}$ around $z$ as illustrated in Fig. 12c.

To obtain the Rashba- and Dresselhaus contributions the spin-galvanic effect is measured for a fixed orientation of $\boldsymbol{S}_{\|}$for all accessible directions $\Theta$ (see Fig. 12c). According to Eq. (19) the current $\boldsymbol{j}_{\mathrm{R}}$ always flows perpendicularly to the spin polarization $\boldsymbol{S}_{\|}$, and $\boldsymbol{j}_{\mathrm{D}}$ encloses an angle $-2 \phi$ with $\boldsymbol{S}_{\|}$. Here $\phi$ is the angle between $\boldsymbol{S}_{\|}$and the $x^{\prime \prime} \|[100]$-axis. Then, the current component along any direction given by the angle $\Theta$ can be written as a sum of the projections of $\boldsymbol{j}_{\mathrm{R}}$ and $\boldsymbol{j}_{\mathrm{D}}$ on this direction

$$
j_{\mathrm{SGE}}(\Theta)=j_{\mathrm{D}} \cos (\Theta+\phi)+j_{\mathrm{R}} \sin (\Theta-\phi) .
$$

Evaluating measurements using this equation yields immediately the ratio between Rashba and Dresselhaus terms. Three directions of spin population $\boldsymbol{S}_{\|}$are particularly suited to extract the ratio between Rashba and Dresselhaus terms. In the first geometry sketched in the left plate of Fig. 13, the spin polarization $\boldsymbol{S}_{\|}$ is set along [100] $(\phi=0)$. Then it follows from Eq. (21) that the currents along the [100]-direction $(\Theta=0)$ and [010]-direction $(\Theta=\pi / 2)$ are equal to $j_{\mathrm{D}}$ and $j_{\mathrm{R}}$, respectively, as shown on the left hand side of Fig. 13. The ratio of Rashba and Dresselhaus currents can be directly read off from the right hand side of Fig. $13, j_{\mathrm{R}} / j_{\mathrm{D}}=j(\pi / 2) / j(0)$. The best value obtained by this method in [21] is $j_{\mathrm{R}} / j_{\mathrm{D}}=\beta_{\mathrm{SIA}} / \beta_{\mathrm{BIA}}=2.1$ in good agreement to theoretical results [66] which predict a dominating Rashba spin-orbit coupling for InAs QWs.

In the second procedure a non-equilibrium spin polarization is induced along [110] $(\phi=\pi / 4)$ or [110] $(\phi=-\pi / 4)$ resulting in the maximum value $j=j_{\mathrm{R}}-j_{\mathrm{D}}$ or $j_{\mathrm{R}}+j_{\mathrm{D}}$, respectively. These values also allow a straight forward determination of $j_{\mathrm{R}} / j_{\mathrm{D}}=\beta_{\mathrm{SIA}} / \beta_{\mathrm{BIA}}$. 


\section{Summary}

A non-equilibrium uniform spin polarization obtained by optical orientation drives an electric current in QWs if they belong to a gyrotropic crystal class. Two different microscopic mechanisms of spin photocurrents can be distinguished, the CPGE and the spin-galvanic effect. In the first effect the coupling of the helicity of light to spin polarized final states with a net linear momentum is caused by selection rules together with band splitting in $\boldsymbol{k}$-space. The current flow in the second effect is driven by asymmetric spin relaxation of a homogeneous non-equilibrium spin polarization. Spin photocurrents are not limited to 2D structures. Most recently they have been predicted for 1D systems like carbon nanotubes of spiral symmetry [67].

Macroscopic measurements of photocurrents in different geometric configurations of experiments allow to conclude on details of the microscopic tensorial spin-orbit interaction [4]. In particular, the relation between the Dresselhaus-like terms and the Rashba term, respectively, may be obtained. Furthermore, the macroscopic in-plane symmetry of QWs may easily be determined. Spin photocurrents were also applied to investigate the mechanism of spin relaxation at monopolar spin orientation [26] where only one type of charge carriers is involved in the excitation-relaxation process. This condition is close to that of electric spin injection in semiconductors. Two methods were applied to determine spin relaxation times: the Hanle effect in the spin-galvanic current [3] and spin sensitive bleaching of photogalvanic currents [22, 23]. The CPGE has also been applied to detect the state of polarization of terahertz radiation with a high time resolution [61].

\section{Acknowledgments}

Financial support by the DFG is gratefully acknowledged.

\section{References}

[1] Semiconductor Spintronics and Quantum Computation, Eds. D.D. Awschalom, D. Loss, N. Samarth, in the series Nanoscience and Technology, Eds. K. von Klitzing, H. Sakaki, R. Wiesendanger, Springer, Berlin 2002.

[2] Y.A. Bychkov, E.I. Rashba, Pis'ma ZhETF 39, 66 (1984) [Sov. JETP Lett. 39, 78 (1984)].

[3] S.D. Ganichev, E.L. Ivchenko, V.V. Bel'kov, S.A. Tarasenko, M. Sollinger, D. Weiss, W. Wegscheider, W. Prettl, Nature (London) 417, 153 (2002).

[4] S.D. Ganichev, W. Prettl, topical review, J. Phys., Condens. Matter 15, R935 (2003).

[5] E.L. Ivchenko, Optical Spectroscopy of Semiconductor Nanostructures, Alpha Science Int., Harrow, UK 2005.

[6] S.D. Ganichev, E.L. Ivchenko, S.N. Danilov, J. Eroms, W. Wegscheider, D. Weiss, W. Prettl, Phys. Rev. Lett. 86, 4358 (2001). 
[7] J. Hübner, W.W. Rühle, M. Klude, D. Hommel, R.D.R. Bhat, J.E. Sipe, H.M. van Driel, Phys. Rev. Lett. 90, 216601 (2003).

[8] M.J. Stevens, A.L. Smirl, R.D.R. Bhat, A. Najimaie, J.E. Sipe, H.M. van Driel, Phys. Rev. Lett. 90, 136603 (2003).

[9] S.D. Ganichev, Petra Schneider, V.V. Bel'kov, E.L. Ivchenko, S.A. Tarasenko, W. Wegscheider, D. Weiss, D. Schuh, D.G. Clarke, M. Merrick, B.N. Murdin, P. Murzyn, P.J. Phillips, C.R. Pidgeon, E.V. Beregulin, W. Prettl, Phys. Rev. B, Rapid Commun. 68, 081302 (2003).

[10] I. Z̆utić, J. Fabian, S. Das Sarma, Phys. Rev. Lett. 88, 066603 (2002).

[11] V.V. Bel'kov, S.D. Ganichev, E.L. Ivchenko, S.A. Tarasenko, W. Weber, S. Giglberger, M. Olteanu, P. Tranitz, S.N. Danilov, Petra Schneider, W. Wegscheider, D. Weiss, W. Prettl, J. Phys. C, Condens. Matter. 17, 3405 (2005).

[12] A.G. Aronov, Yu. B. Lyanda-Geller, Pis'ma ZhETP 50, 398 (1989) [Sov. JETP Lett. 50, 431 (1990)].

[13] V.M. Edelstein, Solid State Commun. 73, 233 (1990).

[14] S.D. Ganichev, S.N. Danilov, Petra Schneider, V.V. Bel'kov, L.E. Golub, W. Wegscheider, D. Weiss, W. Prettl, cond-mat/0403641 (2004).

[15] S.D. Ganichev, S.N. Danilov, Petra Schneider, V.V. Bel'kov, L.E. Golub, W. Wegscheider, D. Weiss, W. Prettl, J. Magn. Magn. Mater., to be published.

[16] A.Yu. Silov, P.A. Blajnov, J.H. Wolter, R. Hey, K.H. Ploog, N.S. Averkiev, Appl. Phys. Lett. 85, 5929 (2004).

[17] Y.K. Kato, R.C. Myers, A.C. Gossard, D.D. Awschalom, Phys. Rev. Lett. 93, 176601 (2004).

[18] E.L. Ivchenko, G.E. Pikus, Pis'ma Zh. Eksp. Teor. Fiz. 27, 640 (1978) [JETP Lett. 27, 604 (1978)].

[19] E.L. Ivchenko, G.E. Pikus, Izv. Akad. Nauk SSSR (Ser. Fiz.) 47, 2369 (1983) [Bull. Acad. Sci. USSR, Phys. Ser. 47, 81 (1983)].

[20] W. Zawadzki, P. Pfeffer, Semicond. Sci. Technol. 19, R1 (2004).

[21] S.D. Ganichev, V.V. Bel'kov, L.E. Golub, E.L. Ivchenko, Petra Schneider, S. Giglberger, J. Eroms, J. DeBoeck, G. Borghs, W. Wegscheider, D. Weiss, W. Prettl, Phys. Rev. Lett. 92, 256601 (2004).

[22] S.D. Ganichev, S.N. Danilov, V.V. Bel'kov, E.L. Ivchenko, M. Bichler, W. Wegscheider, D. Weiss, W. Prettl, Phys. Rev. Lett. 88, 057401-1 (2002).

[23] P. Schneider, J. Kainz, S.D. Ganichev, V.V. Bel'kov, S.N. Danilov, M.M. Glazov, L.E. Golub, U. Rössler, W. Wegscheider, D. Weiss, D. Schuh, W. Prettl, J. Appl. Phys. 96, 420 (2004).

[24] Optical Orientation, Eds. F. Meier, B.P. Zakharchenya, Elsevier Science Publ., Amsterdam 1984.

[25] S.A. Tarasenko, E.L. Ivchenko, V.V. Bel'kov, S.D. Ganichev, D. Schowalter, Petra Schneider, M. Sollinger, W. Prettl, V.M. Ustinov, A.E. Zhukov, L.E. Vorobjev, cond-mat/0301393 (2003). 
[26] S.A. Tarasenko, E.L. Ivchenko, V.V. Bel'kov, S.D. Ganichev, D. Schowalter, Petra Schneider, M. Sollinger, W. Prettl, V.M. Ustinov, A.E. Zhukov, L.E. Vorobjev, J. Supercond. 16, 419 (2003).

[27] E.L. Ivchenko, S.A. Tarasenko, Zh. Eksp. Teor. Fiz. 126, 476 (2004) [JETP 99, 379 (2004)].

[28] S.D. Ganichev, I.N. Yassievich, W. Prettl, J. Phys., Condens. Matter 14, R1263 (2002).

[29] S.D. Ganichev, W. Prettl, Intense Terahertz Excitation of Semiconductors, Oxford University Press, to be published in 2005.

[30] G.M.H. Knippels, X. Yan, A.M. MacLeod, W.A. Gillespie, M. Yasumoto, D. Oepts, A.F.G. van der Meer, Phys. Rev. Lett. 83, 1578 (1999).

[31] S.D. Ganichev, E.L. Ivchenko, H. Ketterl, W. Prettl, L.E. Vorobjev, Appl. Phys. Lett. 77, 3146 (2000).

[32] B.I. Sturman, V.M. Fridkin, The Photovoltaic and Photorefractive Effects in Non-Centrosymmetric Materials, Gordon and Breach Science Publ., New York 1992.

[33] E.L. Ivchenko, G.E. Pikus, Superlattices and Other Heterostructures. Symmetry and Optical Phenomena, Springer, Berlin 1997.

[34] M.I. D'yakonov, V.Yu. Kachorovskii, Fiz. Tekh. Poluprovodn. 20, 178 (1986) [Sov. Phys. Semicond. 20, 110 (1986)].

[35] O. Krebs, P. Voisin, Phys. Rev. Lett. 77, 1829 (1996).

[36] S.D. Ganichev, E.L. Ivchenko, W. Prettl, Physica E 14, 166 (2002).

[37] L.E. Golub, Phys. Rev. B 67, 235320 (2003).

[38] V.V. Belkov, S.D. Ganichev, Petra Schneider, C. Back, M. Oestreich, J. Rudolph, D. Hägele, L.E. Golub, W. Wegscheider, W. Prettl, Solid State Commun. 128, 283 (2003).

[39] M. Sasaki, Y. Ohno, H. Ohno, unpublished.

[40] S.D. Ganichev, V.V. Bel'kov, Petra Schneider, E.L. Ivchenko, S.A. Tarasenko, D. Schuh, W. Wegscheider, D. Weiss, W. Prettl, Phys. Rev. B 68, 035319 (2003).

[41] S.D. Ganichev, M. Sollinger, W. Prettl, D.R. Yakovlev, P. Grabs, G. Schmidt, L. Molenkamp, E.L. Ivchenko, Verhandl. DPG (VI) 36, 1/170 (2001).

[42] W. Weber, S.D. Ganichev, V.V. Belkov, L.E. Golub, W. Prettl, Z.D. Kvon, Hyun-Ick Cho, Jung-Hee Lee, to be published.

[43] S.D. Ganichev, U. Rössler, W. Prettl, E.L. Ivchenko, V.V. Bel'kov, R. Neumann, K. Brunner, G. Abstreiter, Phys. Rev. B 66, 75328 (2002).

[44] N.S. Averkiev, L.E. Golub, M. Willander, J. Phys., Condens. Matter 14, R271 (2002).

[45] N.S. Averkiev, M.I. D'yakonov, Fiz. Tekh. Poluprovodn. 17, 629 (1983) [Sov. Phys. Semicond. 17, 393 (1983)].

[46] A.A. Bakun, B.P. Zakharchenya, A.A. Rogachev, M.N. Tkachuk, V.G. Fleisher, Pis'ma ZhETF 40, 464 (1984) [Sov. JETP Lett. 40, 1293 (1984)].

[47] I. Zuutić, J. Fabian, S. Das Sarma, Appl. Phys. Lett. 79, 1558 (2001).

[48] A.V. Andrianov, I.D. Yaroshetskii, Pis'ma Zh. Eksp. Teor. Fiz. 40, 131 (1984) [Sov. Phys. JETP 40, 882 (1984)]. 
[49] L.I. Magarill, A.M. Palkin, V.N. Sozinov, V.M. Entin, JETP 97, 533 (1990).

[50] L.I. Magarill, Fiz. Tverd. Tela 32, 3558 (2090) [Sov. Phys. Solid State 32, 2064 (1990)].

[51] A.P. Dmitriev, S.A. Emel'yanov, S.V. Ivanov, P.S. Kop'ev, Ya.V. Terent'ev, I.D. Yaroshetskii, Pis'ma Zh. Eksp. Teor. Fiz. 54, 279 (1991) [Sov. Phys. JETP 54, 273 (1991)].

[52] S.A. Emel'yanov, Ya.V. Terent'ev, A.P. Dmitriev, B.Ya. Mel'tser, Pis'ma Zh. Eksp. Teor. Fiz. 68, 768 (1998) [Sov. Phys. JETP 68, 810 (1998)].

[53] V.V. Bel'kov, S.D. Ganichev, Petra Schneider, S. Giglberger, E.L. Ivchenko, S.A. Tarasenko, W. Wegscheider, D. Weiss, W. Prettl, cond- mat/0311474 (2003).

[54] A.A. Gorbatsevich, V.V. Kapaev, Yu.V. Kopaev, Pis'ma Zh. Eksp. Teor. Fiz. 57, 565 (1993) [JETP Lett. 57, 580 (1993)].

[55] O.V. Kibis, Physica E 12, 741 (2002).

[56] E.L. Ivchenko, B. Spivak, Phys. Rev. B 66, 155404 (2002).

[57] Yu.A. Aleshchenko, I.D. Voronova, S.P. Grishechkina, V.V. Kapaev, Yu.V. Kopaev, I.V. Kucherenko, V.I. Kadushkin, S.I. Fomichev, Pis'ma Zh. Eksp. Teor. Fiz. 58, 377 (1993) [JETP Lett. 58, 384 (1993)].

[58] I.V. Kucherenko, L.K. Vodop'yanov, V.I. Kadushkin, Fiz. Tekh. Poluprovodn. 31, 872 (1997) [Semiconductors 31, 740 (1997)].

[59] E.L. Ivchenko, G.E. Pikus, Izv. Akad. Nauk SSSR (Ser. Fiz.) 47, 2369 (1983) [Bull. Acad. Sci. USSR, Phys. Ser. 47, 81 (1983)].

[60] V.I. Belinicher, Fiz. Tverd. Tela 24, 15 (1982) [Sov. Phys. Solid State 24, 7 (1982)].

[61] S.D. Ganichev, H. Ketterl, W. Prettl, Int. J. Infrared Millimeter Waves 24, 847 (2003)

[62] S.A. Tarasenko, N.S. Averkiev, Pis'ma ZhETF 75, 669 (2002) [JETP Lett. 75, $552(2002)]$.

[63] W. Knap, D. Bertho, F. Kobbi, J.L. Robert, G.E. Pikus, F.G. Pikus, Phys. Rev. $B$ 53, 3912 (1996).

[64] N.S. Averkiev, L.E. Golub, Phys. Rev. B 60, 15582 (1999).

[65] J. Schliemann, J.C. Egues, D. Loss, Phys. Rev. Lett. 90, 146801 (2003).

[66] G. Lommer, F. Malcher, U. Rössler, Phys. Rev. Lett. 60, 728 (1988).

[67] E.L. Ivchenko, B. Spivak, Phys. Rev. B 66, 155404-1 (2002). 